\title{
Microfitoplâncton na Baía de Todos Os Santos (Brasil): Composição, diversidade e abundância em um curto período de tempo
}

Lorena Petersen Nascimento Santos, Helen Michelle de Jesus Affe e José Marcos de Castro Nunes

Laboratório de Algas Marinhas (LAMAR), Universidade Federal da Bahia (UFBA), Instituto de Biologia, Rua Barão de Jeremoabo s/n. Ondina, 40170-115, Salvador, Bahia, Brazil

\section{Correspondencia}

José Marcos de Castro Nunes

e-mail: jmcnunes@ufba.br.

Recibido: 29 marzo 2019

Aceptado: 27 marzo 2020

Publicado on-line: 11 mayo 2020

Editado por: María Altamirano Jeschke

\section{RESUMO}

No presente trabalho apresentamos a caracterização da assembleia microfitoplanctônica na Baía de Todos os Santos, considerando as condições abióticas locais, em uma curta série temporal, com o objetivo de ampliar o conhecimento sobre os padrões de composição, diversidade e distribuição do fitoplâncton na baía. Foram realizadas amostragens em três dias consecutivos, no mês de setembro 2016, em cinco pontos distribuídos no setor nordeste da baía. Foram medidos in situ a temperatura, salinidade, oxigênio dissolvido e transparência da água. Amostras foram coletadas utilizando-se garrafa de Van Dorn (1L), em subsuperfície $(\sim 0,5 \mathrm{~m})$, para análises quantitativas do microfitoplâncton. Amostras de $20 \mathrm{~mL}$ foram coletadas por meio de arrastos horizontais de rede de plâncton de $20 \mu \mathrm{m}$ de abertura de malha, para análises de composição da comunidade. Os taxa foram identificados com base em literatura especializada e as análises quantitativas foram realizadas segundo o método de Utermöhl. As variáveis abióticas evidenciaram um ambiente homogêneo, com uma massa d'água quente e salina, tipicamente marinha. Foram identificados 104 táxons e as diatomáceas apresentaram a maior riqueza e abundância de espécies, demonstrando um padrão típico encontrado em sistemas estuarinos tropicais. Considerando a importância da composição e estrutura da comunidade fitoplanctônica para o equilíbrio ecológico da baía e manutenção dos serviços ecossistêmicos, destacamos a ocorrência de espécies potencialmente nocivas, capazes de provocar danos ao sistema em caso de florações, a exemplo do que já ocorreu na ocasião da floração de Akashiwo sanguinea no ano de 2007.

Palavras-chave: Baía de Todos os Santos, diatomáceas, espécies potencialmente nocivas, microalgas marinhas, Salvador - Bahia

\section{ABSTRACT}

Microphytoplancton in the Baía de Todos Os Santos (Brazil): Composition, diversity and abundance in a short period of time

In this work we present the characterization of the microphytoplankton assembly in the Baía de Todos os Santos, considering the local abiotic conditions, in short time series, in order to increase the knowledge about the composition, diversity and distribution patterns of phytoplankton in the bay. Samples were taken on three consecutive days, September 2016, in five points distributed in the northeast sector of the bay. The temperature, salinity, dissolved oxygen and water transparency were measured in situ. Samples (1L) were collected in subsurface $(\sim 0.5 \mathrm{~m})$, for microphytoplankton quantitative analyzes. The species were identified based on specialized literature and quantitative analyzes were performed using the Utermöhl method. The abiotic variables evidenced a homogeneous environment, with a mass of hot and saline water, typically marine. A total of 104 taxa were identified, with diatoms showing the highest species richness and abundance, demonstrating a typical pattern found in tropical estuarine systems. Considering the importance of the composition and structure of the phytoplankton community for the ecological balance of the bay and the maintenance of ecosystem services, we highlight the occurrence of potentially harmful species, capable of causing damage to the system in the event of blooms, such as occurred at the time of bloom of Akashiwo sanguinea in the year 2007.

Key words: Todos os Santos Bay, diatoms, potentially harmful species, marine microalgae, Salvador - Bahia 


\section{INTRODUÇÃO}

O fitoplâncton corresponde ao grupo mais importante de produtores primários marinhos $\mathrm{e}$ estuarinos, respondendo por mais da metade da produtividade primária global (Reynolds, 2006; Falkowski \& Raven, 2007; Brierley, 2017). Seus padrões de composição, diversidade, abundância e distribuição são amplamente influenciados pela variabilidade de fatores ambientais, que modulam assim, as taxas de produtividade primária nos diferentes sistemas (Attrill \& Rundle, 2002; Cloern et al., 2014). Particularmente em ambientes estuarinos, os movimentos de maré e descargas fluviais, condicionam frequentes variações nas demais condições abióticas, com efeitos nas comunidades fitoplanctônicas (Cloern, 1991; Blauw et al., 2012).

Embora estudada desde 1916, quando Zimmermann publicou os primeiros estudos sobre as diatomáceas da região (Gomes et al., 2012), a composição e diversidade da comunidade fitoplanctônica na Baía de Todos os Santos (BTS) persiste como uma lacuna de conhecimento, uma vez que a grande maioria dos estudos na área encontram-se sob forma de dados não publicados, em trabalhos de conclusão de curso. Um estudo de longo prazo sobre a variabilidade intra-anual (20122014) da oceanografia da Baía de Todos os Santos apresenta as diatomáceas como grupo mais abundante na comunidade fitoplanctônica, seguidas pelos dinoflagelados e cianobactérias, respectivamente, apresentando composição típica de ambientes estuarino-marinhos (Lessa et al., 2018).

Com uma área de $1223 \mathrm{~km}^{2} \mathrm{e}$ profundidade média de $9,8 \mathrm{~m}$ a BTS (12050 S 383 W) é um sistema estuarino típico (Cirano \& Lessa, 2007; Lessa et al., 2009), figurando como a maior baía do litoral brasileiro. O clima da região é tropical úmido, com temperatura média anual de $25,2{ }^{\circ} \mathrm{C}$ e precipitação média anual de $2.100 \mathrm{~mm}$ (Cirano \& Lessa, 2007; Hatje \& Andrade, 2009). A circulação interna na baía é forçada por marés, de regime semi-diurno, que somada ao padrão de circulação sazonal do vento, principal forçante nas camadas superficiais, condicionam uma coluna d'água bem misturada e com características oceânicas (Wolgemuth et al., 1981; Lessa et al., 2009; Santana et al., 2018). A influência da água Tropical, caracterizada por temperaturas acima de $20 \stackrel{\circ}{\circ} \mathrm{C}$ e salinidade acima de $36 \%$, é marcante na baía ao longo de todo o ano, especialmente no período mais seco (setembro a janeiro) (Cirano \& Lessa, 2007; Lessa et al., 2009).

A bacia de drenagem da baía é composta, principalmente, pelos rios Paraguaçu, Subaé e Jaguaribe, que juntos contribuem com uma descarga média de $120 \mathrm{~m}^{3} / \mathrm{s}$ no sistema (Hatje \& Andrade, 2009). Abriga ecossistemas de fundamental importância ecológica, compreendendo um complexo de pequenas baías internas, ilhas, recifes de coral, manguezais e estuários, além do destaque econômico (e.g. terminais portuários, intensas atividades turísticas) fornecendo uma série de serviços ecossistêmicos essenciais para uma população superior a três milhões de habitantes concentrada em seu entorno (Hatje \& Andrade, 2009). As populações ribeirinhas tradicionais têm sua subsistência baseada na pesca e mariscagem (Hydros, 2005; SEAP, 2007) atividades que dependem diretamente da produtividade das microalgas fitoplanctônicas (Cirano \& Lessa, 2007; Hatje \& Andrade 2009; Soares et al., 2011).

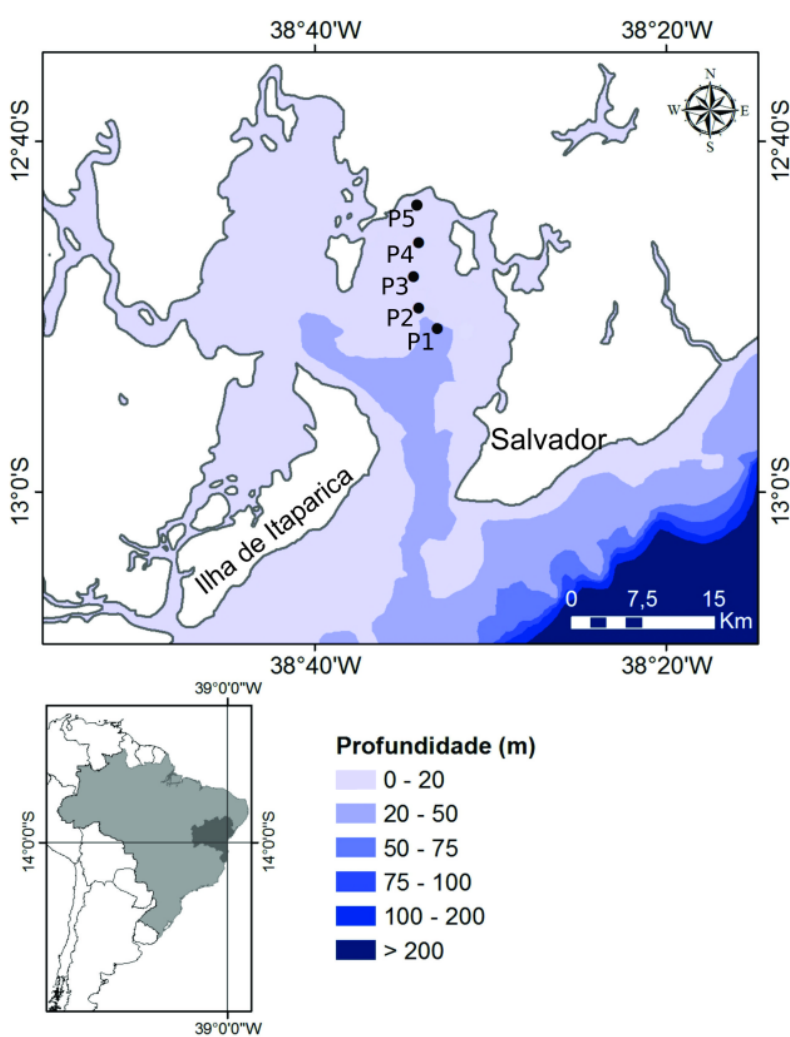

Figura 1. Mapa da região estudada mostrando os pontos de coleta (P1, P2, P3, P4, P5).

Figure 1. Map of the studied region showing collection points (P1, P2, P3, P4, P5).

Os estudos desenvolvidos na BTS estão principalmente concentrados na região de influência do Porto de Aratu e demonstraram que mudanças nas condições abióticas (i.e. temperatura, oxigênio dissolvido, salinidade e $\mathrm{pH}$ ), refletem alterações temporais na composição e biomassa fitoplanctônicas (Ferreira et al., 2012; Forte Neto et al., 2014; Lessa et al., 2018). O evento mais marcante ocorreu em 2007, quando após um período de alta pluviosidade, que condicionou uma grande descarga de nutrientes dissolvidos na baía, proveniente da bacia de drenagem do Rio Paraguaçu, registrou-se uma enorme floração algal nociva da espécie Akashiwo sanguínea (Hirasaka) G. Hansen \& Moestrup (Dinophyceae). As elevadas biomassa e densidade celulares desse dinoflagelado não tóxico provocaram condições de anoxia na coluna d'água, além de obstruir as brânquias, ocasionando a mortandade de mais de sete toneladas de peixes (Argôlo \& Carmel, 2007). 
No presente trabalho apresentamos a caracterização da assembleia microfitoplanctônica no setor norte da Baía de Todos os Santos, considerando as condições abióticas locais, em 3 dias consecutivos, com o objetivo de ampliar 0 conhecimento sobre os padrões de composição, diversidade e distribuição do fitoplâncton na baía em uma curta série temporal.

\section{MATERIAL E MÉTODOS}

As amostras foram coletadas em setembro de 2016, em cinco pontos (amostrados em triplicata), em subsuperfície ( 0,5 $\mathrm{m}$ de profundidade), na região nordeste da baía (Figura 1), em 3 dias consecutivos. Para caracterização abiótica da área de estudos foram medidos in situ, ao longo de todo o período de amostragem, a temperatura e salinidade, utilizando-se uma sonda multi-parâmetros (Horiba@). Além da transparência da água, estimada pelas medidas de profundidade de desaparecimento do disco de Secchi. Os dados de pluviosidade foram obtidos no site da SUDENE/INPE (http://proclima.cptec.inpe.br) considerando-se o acumulado dos 30 dias que precederam o período de amostragem.

As análises da composição do microfitoplâncton foram realizadas a partir de amostras (200 mL) coletadas utilizando-se rede de plâncton, com abertura de malha de $20 \mu \mathrm{m}$, por meio de arrastos horizontais (5 minutos de duração), em cada ponto (15 amostras/dia), com o barco desenvolvendo velocidade aproximada de 3 nós. As amostras (500 $\mathrm{mL}$ ) para análise da densidade celular (cél $\mathrm{L}^{-1}$ ) foram coletadas, utilizando-se garrafa de Van Dorn (15 amostras/dia). Todas as amostras foram armazenadas em frascos escuros de polietileno, fixadas com lugol (1\%).

A identificação das espécies foi realizada por meio de observações de lâminas rápidas, com aumentos de 200x a 400x, ao microscópio direto (Olympus $\AA$ ), com base em bibliografia especializada (e.g. Cupp, 1943; Balech, 1988; Tomas, 1997; Throndsen et al., 2007). A análise quantitativa do fitoplâncton (cel $\mathrm{L}^{-1}$ ), foi realizada segundo o método de Utermöhl (1958), em câmaras de sedimentação (100 mL), com tempo de sedimentação de 24h, contando-se as células microfitoplanctônicas (20$200 \mu \mathrm{m})$, em todo o fundo da câmara, ao microscópio invertido (Motic AE 2000), com aumento de 200x a 400x.

Os parâmetros ambientais foram analisados aplicando-se a análise de variância de KruskalWallis, após verificados o não atendimento dos pressupostos para análises paramétricas (normalidade e homocedasticidade) utilizando os testes de Shapiro-Wilk e Levene, respectivamente. Em seguida aplicou-se o teste de comparação múltipla de valores $\mathrm{p}(\mathrm{p}<0.05)$, para verificar a ocorrência de diferenças significativas das variáveis ambientais entre os pontos de coleta e durante o período de amostragem.

A estrutura da comunidade foi analisada usando as métricas ecológicas: riqueza de espécies (S), diversidade de Shannon-Weaver $\left(H^{\prime}\right)$ e equabilidade de Pielou (J'), considerando a composição e densidade celular em cada ponto de coleta.
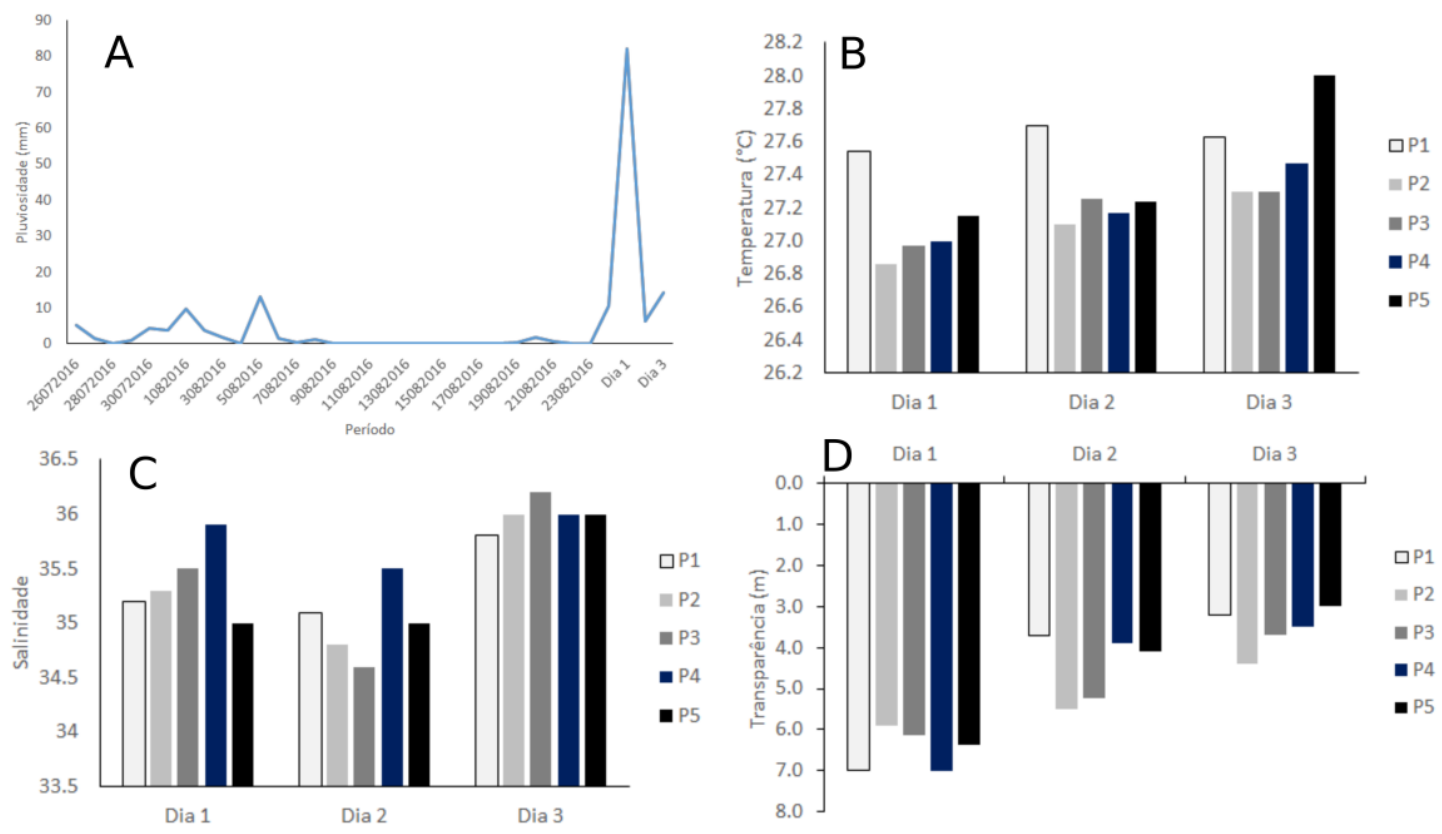

Figura 2. (A) Precipitação pluviométrica $(\mathrm{mm})$ diária nos trinta dias anteriores ao período de coleta (26/07/16 a 24/08/16) e nos três dias de coleta (25-27/08/16); $(B)$ temperatura da água $\left({ }^{\circ} \mathrm{C}\right)$, (C) salinidade $(\%)$ e $(\mathrm{D})$ transparência na coluna d'água $(\mathrm{m})$, registradas nos três dias de coleta (25-27/08/16) na Baía de Todos os Santos.

Figure 2. (A) Daily precipitation $(\mathrm{mm})$ in the thirty days prior to the sampling period (07/26/2016 to 08/24/2016), and during the three sampling days (25-27/08/2016); (B) water temperature $\left({ }^{\circ} \mathrm{C}\right)$, (C) salinity (\%o) and (D) transparency in the water column $(m)$, recorded on the three sampling days (25-27/08/2016) at Baía de Todos os Santos. 


\section{RESULTADOS E DISCUSSÃO}

Foram identificados 104 táxons, dos quais 65 pertencentes a classe Bacillariophyceae, 25 a Dinophyceae, 4 a Cyanophyceae, 3 a Euglenophyceae, 3 a Coccolithophyceae, e um táxon em cada uma das classes: Chlorophyceae, Conjugatophyceae, Dictyochophyceae e Prymnesiophyceae (Tabela 1). De maneira geral, as zonas costeiras, estuarinas e a plataforma continental brasileira são tipicamente dominadas pelos grupos das diatomáceas, seguido pelos dinoflagelados (Rezende et al., 2015; Carvalho et al., 2016; Affe et al., 2018). Na BTS, as diatomáceas constituem o principal grupo do microfitoplâncton, ocorrendo em altas taxas de abundância nos diversos setores da baía especialmente durante 0 período chuvoso (Lopes et al., 2009; Ferreira et al., 2012).

A maior riqueza (77 táxons) foi registrada no dia 2 de coleta, e a menor (55 táxons) no dia 3. A diversidade de espécies variou de 1,7 bits.cel ${ }^{-1}$ no dia 3 a 2,8 bits.cel ${ }^{-1}$ no dia1 (Tabela 2), com valores de equabilidade entre 0,6 no dia 3 e 0,8 nos dias 1 e 2 (Tabela 2). Durante período de monitoramento na baía (2013-2014), as diatomáceas dominaram a comunidade fitoplanctônica (73\% a $85 \%)$, seguidas dos dinoflagelados (4\% a $10 \%)$ e as cianobactérias
(2\% a 24\%) (Lessa et al., 2018). Esse mesmo padrão foi registrado ao longo dos três dias de análise do presente estudo, quando a densidade celular do microfitoplâncton variou de $7,52 \times 10^{3}$ cél

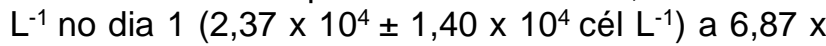
$10^{4}$ cél $L^{-1}$ no dia $3\left(3,38 \times 10^{4} \pm 2,05 \times 10^{4}\right.$ cél L-1 $^{-1}$ (Tabela 1). Observando que, no dia 3 foram registras as menores diversidade e equabilidade e a maior densidade celular, mas sem diferenças significativas $(p=0,03)$ ao longo de todo o período.

A espécie Coscinodiscus wailesii Gran \& Angst ocorreu em todos os pontos de coleta, nos três dias de estudo, apresentando taxa de densidade variando entre $2,84 \times 10^{3}$ cél L-1 $\left(6,34 \pm 3,62 \times 10^{3}\right.$ cél $\left.\mathrm{L}^{-1}\right)$ no dia 1 , a $1,42 \times 10^{4}$ cél L-1 $^{-1}(9,52 \pm 4,60 \times$

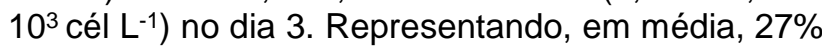
$( \pm 0,5 \%)$ da abundância total de espécies nas amostras. Trata-se de uma espécie marinha invasora nos sistemas costeiros brasileiros, que vem sendo registrada em algumas regiões da costa, chegando a representar até $90 \%$ da abundância do microfitoplâncton (Fernandes et al., 2001). Esta é considerada uma espécie potencialmente nociva devido a sua capacidade de produzir elevada biomassa, podendo causar danos aos peixes e invertebrados marinhos, além de provocar alterações estéticas na coluna d'água pela intensa produção de mucilagem (Castro \& Moser, 2010).

Tabela 1. Inventário florístico do microfitoplâncton nos três dias de coleta (25, 26, 27 agosto 2016), nos pontos de amostragem na Baía de Todos os Santos (P1, P2, P3, P4, P5). (X = presença). * Os taxa estão apresentados em ordem alfabética

Table 1. Floristic inventory of the microphytoplankton of the three days of collection (October $25^{\text {th }}, 26^{\text {th }}$ and $27^{\text {th }} 2016$ ), at sampling points at Baía de Todos os Santos (P1, P2, P3, P4, P5). ( $x=$ presence). * taxa are mentioned in alphabetical order.

\begin{tabular}{|c|c|c|c|c|c|c|c|c|c|c|c|c|c|c|c|}
\hline \multirow{2}{*}{ Taxa } & \multicolumn{5}{|c|}{ Dia 1} & \multicolumn{5}{|c|}{ Dia 2} & \multicolumn{5}{|c|}{ Dia 3} \\
\hline & P1 & $\mathbf{P 2}$ & P3 & P4 & P5 & P1 & $\mathbf{P 2}$ & P3 & P4 & P5 & $\mathbf{P 1}$ & P2 & P3 & P4 & P5 \\
\hline $\begin{array}{l}\text { Bacillariophyceae } \\
\text { Achnanthaceae }\end{array}$ & & & & & & & & & & & & & & & \\
\hline $\begin{array}{l}\text { Achnanthes brevipes C. Agardh } \\
\text { Bacillariaceae }\end{array}$ & & & & & & & $\mathrm{X}$ & $\mathrm{X}$ & $\mathrm{X}$ & $\mathrm{X}$ & & $\mathrm{X}$ & $\mathrm{X}$ & $\mathrm{X}$ & $\mathrm{X}$ \\
\hline $\begin{array}{l}\text { Bacillaria paxillifera (O.F.Müller) T. Marsson } \\
\text { Cylindrotheca closterium(Ehrenberg) Reimann }\end{array}$ & $\mathrm{X}$ & & $\mathrm{X}$ & $\mathrm{X}$ & $x$ & $\mathrm{X}$ & & & & $\mathrm{X}$ & $\mathrm{X}$ & & $\mathrm{X}$ & $\mathrm{X}$ & $x$ \\
\hline \& J.C.Lewin & $\mathrm{X}$ & $x$ & & $x$ & $x$ & $x$ & $x$ & $x$ & $x$ & $\mathrm{X}$ & $X$ & $x$ & & $X$ & $x$ \\
\hline Nitzschia longissima (Brébisson) Grunow & $\mathrm{X}$ & $\mathrm{X}$ & $x$ & & $x$ & $\mathrm{X}$ & $\mathrm{X}$ & & & $\mathrm{X}$ & & & & & \\
\hline Nitzschia lorenziana Grunow & & $x$ & $x$ & $\mathrm{X}$ & $x$ & $x$ & $\mathrm{X}$ & $x$ & $x$ & & $\mathrm{X}$ & $x$ & $x$ & $\mathrm{X}$ & \\
\hline Nitzschia reversa W. Smith & $\mathrm{X}$ & $\mathrm{X}$ & & $\mathrm{X}$ & & $x$ & $\mathrm{X}$ & $\mathrm{X}$ & $x$ & & & & & & \\
\hline Nitzschia sigmoidea (Nitzsch) W. Smith & & & & & & $x$ & $x$ & & $x$ & $\mathrm{X}$ & & & & & \\
\hline $\begin{array}{l}\text { Nitzschia sp. } \\
\text { Biddulphiaceae }\end{array}$ & & & & & & & & $\mathrm{X}$ & $\mathrm{X}$ & $\mathrm{X}$ & $x$ & $X$ & $X$ & & $X$ \\
\hline $\begin{array}{l}\text { Biddulphia alternans (Bailey) Van Heurck } \\
\text { Catenulaceae }\end{array}$ & $X$ & $X$ & $x$ & $X$ & $x$ & & & & & & $X$ & $X$ & $X$ & $X$ & $X$ \\
\hline $\begin{array}{l}\text { Amphora sp. } \\
\text { Chaetocerotaceae }\end{array}$ & & & & & & $X$ & $X$ & $X$ & $X$ & & & & & & \\
\hline $\begin{array}{l}\text { Chaetoceros danicus Cleve } \\
\text { Chaetoceros decipiens Cleve } \\
\text { Chaetoceros peruvianus Brightwell } \\
\text { Climacospheniaceae }\end{array}$ & & & & & & $X$ & & $X$ & $X$ & $X$ & & & & & \\
\hline $\begin{array}{l}\text { Climacosphenia moniligera Ehrenberg } \\
\text { Cocconeidaceae }\end{array}$ & $x$ & $x$ & $x$ & $x$ & $x$ & $X$ & & $x$ & $x$ & $x$ & $x$ & $x$ & $x$ & $x$ & $x$ \\
\hline $\begin{array}{l}\text { Cocconeis cf. placentula Ehrenberg } \\
\text { Cocconeis sp. }\end{array}$ & & & & & & $X$ & $X$ & $X$ & $X$ & & & & & & \\
\hline Cocconeis sp. & $X$ & & $x$ & & & & & & & & & & & & \\
\hline
\end{tabular}




\begin{tabular}{|c|c|c|c|c|c|c|c|c|c|c|c|c|c|c|c|}
\hline \multirow{2}{*}{ Taxa } & \multicolumn{5}{|c|}{ Dia 1} & \multicolumn{5}{|c|}{ Dia 2} & \multicolumn{5}{|c|}{ Dia 3} \\
\hline & P1 & P2 & P3 & P4 & P5 & P1 & P2 & P3 & P4 & P5 & P1 & P2 & P3 & P4 & P5 \\
\hline Coscinodiscus wailesii Gran \& Angst & $\mathrm{X}$ & $\mathrm{X}$ & & $\mathrm{X}$ & $\mathrm{X}$ & & $\mathrm{X}$ & $\mathrm{X}$ & $\mathrm{X}$ & $\mathrm{X}$ & $\mathrm{X}$ & $\mathrm{X}$ & $\mathrm{X}$ & $\mathrm{X}$ & $\mathrm{X}$ \\
\hline Coscinodiscus sp. & $X$ & $\mathrm{X}$ & $\mathrm{X}$ & $\mathrm{X}$ & $x$ & $\mathrm{X}$ & $\mathrm{X}$ & $x$ & $x$ & $\mathrm{X}$ & & $\mathrm{X}$ & $\mathrm{X}$ & $\mathrm{X}$ & $\mathrm{X}$ \\
\hline $\begin{array}{l}\text { Palmerina hardmaniana (Grev.) Hasle } \\
\text { Corethraceae }\end{array}$ & $x$ & & $x$ & $\mathrm{X}$ & & & & & & & & & & & \\
\hline $\begin{array}{l}\text { Corethron criophilum Castracane } \\
\text { Cymbellaceae }\end{array}$ & & & & & & $x$ & & $x$ & $x$ & $x$ & & & & & \\
\hline $\begin{array}{l}\text { Cymbella sp. } \\
\text { Diploneidaceae }\end{array}$ & $X$ & & $\mathrm{X}$ & $\mathrm{X}$ & $x$ & & & & & & $\mathrm{X}$ & & $\mathrm{X}$ & $\mathrm{X}$ & $\mathrm{X}$ \\
\hline Diploneis interrupta (Kütz.) Cleve & $x$ & $x$ & & & $x$ & $x$ & $x$ & & $x$ & $x$ & $x$ & $x$ & & $x$ & $x$ \\
\hline $\begin{array}{l}\text { Diploneis cf. didyma (Ehrenberg) Ehrenberg } \\
\text { Fragilariaceae }\end{array}$ & $\mathrm{X}$ & & $\mathrm{X}$ & & $x$ & & $\mathrm{X}$ & $\mathrm{X}$ & $x$ & & & & & & \\
\hline Podocystis adriatica (Kütz.) Ralfs & $x$ & $\mathrm{X}$ & $\mathrm{X}$ & & & & & & & & & & & & \\
\hline $\begin{array}{l}\text { Synedra sp. } \\
\text { Grammatophoraceae }\end{array}$ & $\mathrm{X}$ & $\mathrm{X}$ & $x$ & $x$ & $x$ & $x$ & & $x$ & $x$ & $\mathrm{X}$ & $x$ & $\mathrm{X}$ & $x$ & & $x$ \\
\hline Grammatophora marina (Lyngbye) Kützing & $\mathrm{X}$ & & & $\mathrm{X}$ & $X$ & & & & & & $\mathrm{X}$ & $\mathrm{X}$ & $\mathrm{X}$ & $\mathrm{X}$ & \\
\hline $\begin{array}{l}\text { Grammatophora sp. } \\
\text { Isthmiaceae }\end{array}$ & $\mathrm{X}$ & $\mathrm{X}$ & $\mathrm{X}$ & $\mathrm{X}$ & $\mathrm{X}$ & & & & & & & & & & \\
\hline $\begin{array}{l}\text { Isthmia enervis Ehrenberg } \\
\text { Leptocylindraceae }\end{array}$ & & & $\mathrm{X}$ & $\mathrm{X}$ & $X$ & $\mathrm{X}$ & $\mathrm{X}$ & & $\mathrm{X}$ & $\mathrm{X}$ & & & & & \\
\hline $\begin{array}{l}\text { Leptocylindrus danicus Cleve } \\
\text { Lyrellaceae } \\
\text { Lyrella hennedyi (W.Smith) Stickle \& }\end{array}$ & $\mathrm{X}$ & & $\mathrm{X}$ & $\mathrm{X}$ & $\mathrm{X}$ & & & & & & & & & & \\
\hline D.G.Mann & $\mathrm{X}$ & $\mathrm{X}$ & & $\mathrm{X}$ & $\mathrm{X}$ & $\mathrm{X}$ & $\mathrm{X}$ & $\mathrm{X}$ & & $\mathrm{X}$ & & & & & \\
\hline $\begin{array}{l}\text { Lyrella lyra (Ehrenberg) Karajeva } \\
\text { Lithodesmiaceae }\end{array}$ & $\mathrm{X}$ & $X$ & $\mathrm{X}$ & & $\mathrm{X}$ & $\mathrm{X}$ & $\mathrm{X}$ & $\mathrm{X}$ & & & $\mathrm{X}$ & $\mathrm{X}$ & $\mathrm{X}$ & $x$ & $X$ \\
\hline $\begin{array}{l}\text { Tropidoneis lepidoptera (Gregory) Cleve } \\
\text { Mastogloiaceae }\end{array}$ & & & & & & & & & & & & $x$ & $x$ & $x$ & $x$ \\
\hline $\begin{array}{l}\text { Mastogloia sp. } \\
\text { Melosiraceae }\end{array}$ & & & & & & & $x$ & $x$ & $x$ & $x$ & $x$ & $x$ & & $x$ & $X$ \\
\hline $\begin{array}{l}\text { Melosira moniliformes (O.F. Müller) C. Agardh } \\
\text { Naviculaceae }\end{array}$ & & & & & & $x$ & & $x$ & $x$ & $x$ & & & & & \\
\hline
\end{tabular}

\section{Gyrosigma angulatum (Quekett) Griffith \&}

Henfrey

Gyrosigma balticum (Ehrenberg) Rabenhorst

Gyrosigma littorale (W. Smith) Griffth \&

Henfrey

Gyrosigma sp.

Haslea sp.

Navicula bicapitata O'Meara

Navicula directa (W.Smith) Ralfs

Navicula sp.

Trachyneis aspera (Ehrenberg) Cleve

Trachyneis sp.

Paraliaceae

Paralia sulcata (Ehrenberg) Cleve

Pinnulariaceae

Pinnularia sp.

Plagiotropidaceae

Manguinea sp.

Meuniera membranacea (Cleve) P.C. Silva

Pleurosigmataceae

Donkinia recta (Donkin) Grunow

Pleurosigma angulatum W. Smith

Pleurosigma cf. affine Grunow

Pleurosigma formosum W. Smith

Pleurosigma normanii Ralfs

Pleurosigma sp.

\section{Rhizosoleniaceae}

Guinardia flaccida (Castracane) H. Peragallo

Rhizosolenia acuminata (H.Perag.) H. Perag.

Rhizosolenia decipiens B.G. Sundström

Rhizosolenia imbricata Brightwell

Rhizosolenia sp.

Neocalyptrella robusta (Norman ex Ralfs)

Hern.-Becerril \& Meave Castillo

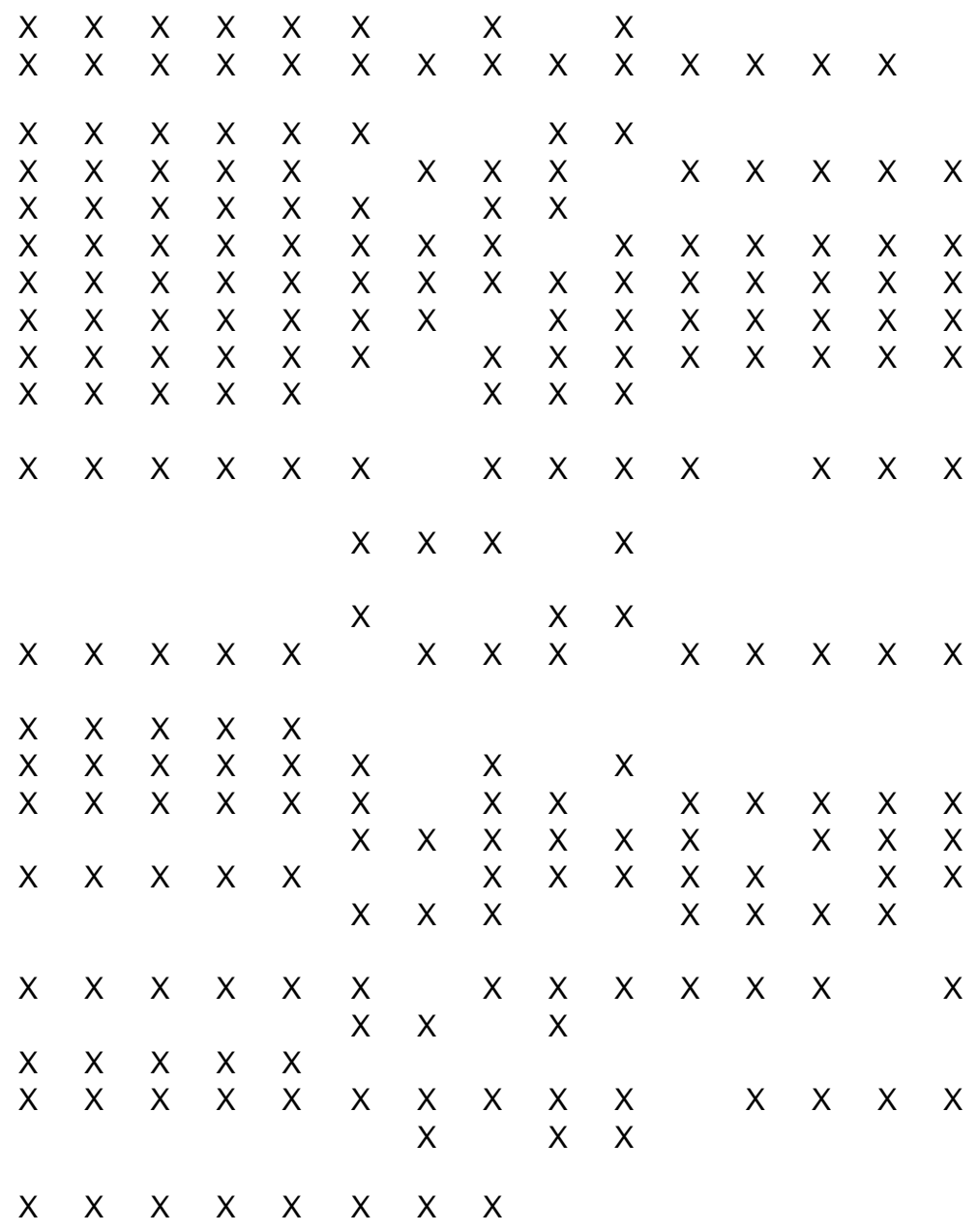




\section{Taxa}

\begin{tabular}{llllllll}
\multicolumn{1}{c}{ Dia 1 } & & & & Dia 2 \\
P1 & P2 & P3 & P4 & P5 & P1 & P2 & P3
\end{tabular}

Dia 3

\section{Stephanodiscaceae}

Cyclotella cf. striata (Kützing) Grunow

Surirellaceae

Surirella fastuosa Ehrenberg

Surirella febigerii Lewis

Petrodictyon gemma (Ehrenb.) D.G. Mann

UInariaceae

Bleakeleya notata (Grunow) Round

Coccolithophyceae

Stephanolithiaceae

Calciosolenia murray Gran

Syracosphaeraceae

Syracosphaera prolongata Gran ex Lohmann

Syracosphaera sp.

\section{Chlorophyceae}

\section{Scenedesmaceae}

Desmodesmus sp.

\section{Conjugatophyceae}

Closteriaceae

Closterium sp.

\section{Cyanophyceae}

\section{Gomontiellaceae}

Komvophoron schmidlei (Jaag) Anagnostidis \&

Komárek

\section{Merismopediaceae}

Merismopedia sp.

Microcoleaceae

Trichodesmium erythraeum Ehrenberg

Pseudoanabaenaceae

Pseudoanabaena sp.

Dictyochophyceae

Dictyochaceae

Dictyocha fíbula Ehrenberg

\section{Dinophyceae}

Brachidiniaceae

Karenia brevis (C.C.Davis) Gert Hansen \& $\varnothing$. Moestrup

\section{Ceratiaceae}

Tripos furca (Ehrenberg) F. Gómez

Tripos furca var. hircus (Schröd.) F. Gómez

Tripos fusus (Ehrenberg) F. Gómez

Tripos macroceros (Ehrenberg) F. Gómez

Tripos trichoceros (Ehrenberg) F. Gómez

Tripos pentagonum Gourret

\section{Dinophysaceae}

Dinophysis caudata Saville-Kent

Dinophysis sp.

\section{Gymnodiniaceae}

Akashiwo sanguinea (K.Hirasaka) G. Hansen \& Moestrup

Gymnodinium fuscum (Ehrenberg) Stein

Gyrodinium falcatum Kof. \& Swezy

Gyrodinium cf. spirale (Bergh) Kofoid \& Swezy

Gyrodinium sp.

Oxytoxaceae

Oxytoxum sp.

Peridiniaceae

Peridinium sp.

\section{Prorocentraceae}

Prorocentrum compressum (J.W.Bailey) Abé ex J.D. Dodge

Prorocentrum micans Ehrenberg

Prorocentrum cf.gracile Schütt

Protoperidiniaceae

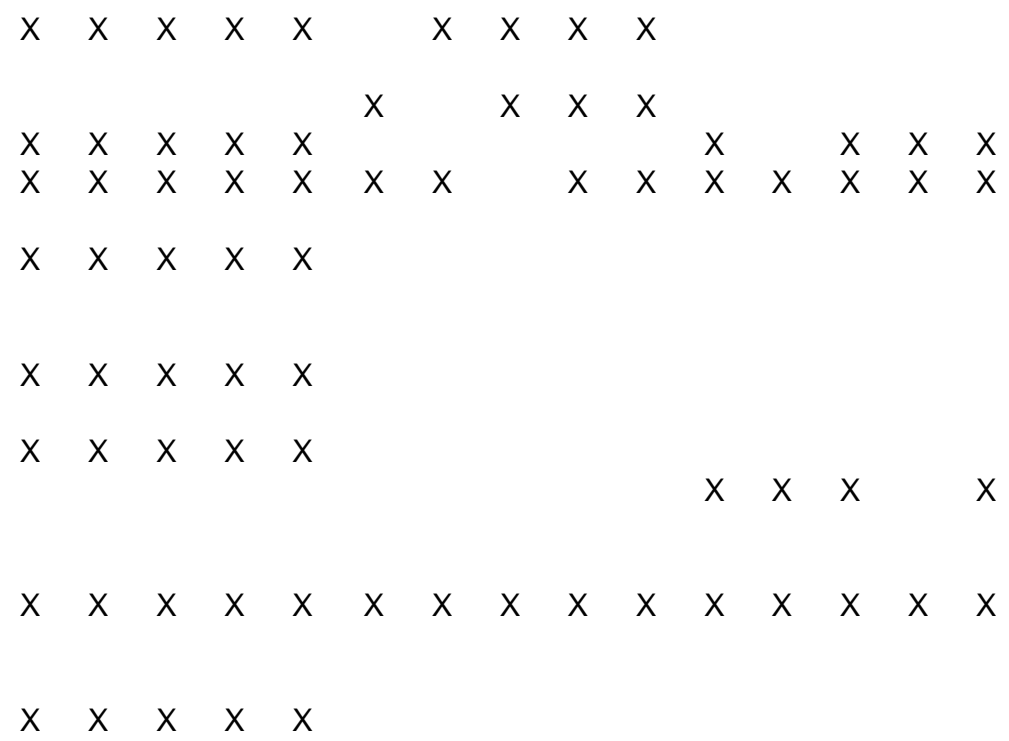




\begin{tabular}{|c|c|c|c|c|c|c|c|c|c|c|c|c|c|c|c|}
\hline \multirow{2}{*}{ Taxa } & \multicolumn{5}{|c|}{ Dia 1} & \multicolumn{5}{|c|}{ Dia 2} & \multicolumn{5}{|c|}{ Dia 3} \\
\hline & P1 & P2 & P3 & P4 & P5 & P1 & P2 & P3 & P4 & P5 & P1 & P2 & P3 & P4 & P5 \\
\hline Protoperidinium brevipes (Paulsen) Balech & $\mathrm{X}$ & $\mathrm{X}$ & $\mathrm{X}$ & $\mathrm{X}$ & $\mathrm{X}$ & & & & & & & & & & \\
\hline Protoperidinium conicum (Gran) Balech & $x$ & $x$ & $x$ & $x$ & $x$ & $\mathrm{X}$ & $x$ & $x$ & $x$ & $x$ & & & & & \\
\hline Protoperidinium pentagonum (Gran) Balech & & & & & & $\mathrm{X}$ & $x$ & $\mathrm{X}$ & $x$ & $\mathrm{X}$ & $x$ & $x$ & $\mathrm{X}$ & $\mathrm{X}$ & $x$ \\
\hline Protoperidinium steinii (Jørgensen) Balech & $x$ & $\mathrm{X}$ & $\mathrm{X}$ & $x$ & $\mathrm{X}$ & $X$ & $\mathrm{X}$ & $X$ & $x$ & $x$ & & & & & \\
\hline $\begin{array}{l}\text { Protoperidinium sp. } \\
\text { Thoracosphaeraceae }\end{array}$ & $x$ & $\mathrm{X}$ & $x$ & $x$ & $x$ & $x$ & $x$ & $x$ & $x$ & $x$ & $x$ & $x$ & $X$ & $\mathrm{X}$ & $x$ \\
\hline $\begin{array}{l}\text { Scrippsiella trochoidea (Stein) Loeblich III } \\
\text { Euglenophyceae } \\
\text { Euglenaceae }\end{array}$ & $\mathrm{X}$ & $\mathrm{X}$ & $x$ & $x$ & $x$ & $x$ & $\mathrm{X}$ & $X$ & $x$ & $x$ & $X$ & $x$ & $\mathrm{X}$ & $X$ & $x$ \\
\hline $\begin{array}{l}\text { Euglena sp. } \\
\text { Eutreptiaceae }\end{array}$ & $x$ & $x$ & $x$ & $x$ & & $x$ & $x$ & $x$ & $x$ & $x$ & $x$ & $x$ & $x$ & $x$ & $x$ \\
\hline $\begin{array}{l}\text { Eutreptia cf. viridis Perty } \\
\text { Phacaceae }\end{array}$ & $x$ & $x$ & $x$ & $x$ & $x$ & $x$ & $x$ & $x$ & $x$ & $x$ & & & & & \\
\hline $\begin{array}{l}\text { Phacus sp. } \\
\text { Prymnesiophyceae } \\
\text { Noelaerhabdaceae } \\
\text { Emiliania huxleyi (Lohmann) W.W. Hay \& H.P. }\end{array}$ & & & & & & & & & & & $x$ & $x$ & $x$ & $x$ & $x$ \\
\hline Mohler & $x$ & 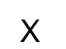 & $x$ & & $x$ & $x$ & & $x$ & $x$ & $x$ & $x$ & $x$ & $x$ & $x$ & $x$ \\
\hline
\end{tabular}

Cabe destacar ainda a ocorrência das espécies Trichodesmium erythraeum Ehrenberg ex Gomont, Akashiwo sanguinea, Kareniabrevis (C.C. Davis) Gert Hansen \& $\varnothing$. Moestrup, Tripos fusus (Ehrenberg) F. Gómez, Gymnodinium sp. e Dinophysis caudata W.S. Kent. Com exceção de $D$. caudata, que é uma espécie potencialmente tóxica (Reguera et al., 2014), todas as demais são classificadas como potencialmente nocivas, pela capacidade de produção de elevada biomassa, capazes de provocar perdas na comunidade de peixes e invertebrados, pelo entupimento de brânquias ou depleção do oxigênio dissolvido na água (Moestrup et al., 2009; Proença et al., 2010). A exemplo de $A$. sanguinea, que já foi relatada causando uma floração nociva na BTS (Saraiva, 2008; Souza et al., 2008) e T. erythraeum, cujas florações têm sido registradas com uma certa frequência na costa Nordeste do Brasil (Satô et al., 1966, Medeiros et al., 1999, Siqueira et al., 2006, Carvalho et al., 2008, Proença et al., 2009; Affe et al., 2016).

Os taxa potencialmente nocivos identificados na BTS, em sua maioria foram registrados em baixas taxas de abundância, a princípio não indicando riscos iminentes na área. Entretanto, considerando a importância da composição e estrutura da comunidade para o equilíbrio ecológico da baía e manutenção dos serviços ecossistêmicos, é importante levar em conta que, mudanças repentinas nas condições abióticas do sistema podem ser favoráveis a floração de uma dessas espécies, a exemplo do que já ocorreu na ocasião da floração de Akashiwo sanguinea. Nesse curto período de tempo observamos aumento na abundância total do microfitoplâncton, principalmente da espécie Coscinodiscus wailesii, após episódio de alta pluviosidade na baía. Destacamos assim, a importância de estudos mais frequentes, incluindo análises de nutrientes inorgânicos dissolvidos, para possível prevenção destes eventos.

Nossas coletas foram realizadas no início da estação seca (setembro 2016), registrando-se uma precipitação acumulada de $61,7 \mathrm{~mm}$, nos trinta dias imediatamente anteriores (Figura 2A).

As variações de temperatura e salinidade na BTS são moduladas por fatores astronômicos como a maré e a sazonalidade climática (Lessa et al., 2018). A temperatura da água apresentou mínima de 26,9 ${ }^{\circ} \mathrm{C}$ no dia $1\left(27,1 \pm 0,2{ }^{\circ} \mathrm{C}\right)$ e máxima de $28,0{ }^{\circ} \mathrm{C}$ no dia $3\left(27,5 \pm 0,2{ }^{\circ} \mathrm{C}\right)$ (Figura $\left.2 \mathrm{~B}\right)$. Não diferindo significativamente $(p=0,68)$ ao longo do período de coleta. Valores típicos para esse período do ano na BTS, quando a temperatura do ar varia entre $21^{\circ} \mathrm{C}$ e $22^{\circ} \mathrm{C}$ (Cirano \& Lessa, 2007). A salinidade variou de $34,6 \%(35 \pm 0,3)$ no dia 2 a $36,2 \%$ o $(36 \pm 0,1)$ no dia 3 (Figura $2 \mathrm{C}$ ), não diferindo significativamente ao longo do período de coleta $(p=0,14)$. Esses valores estão de acordo com os dados de monitoramento da baía $(32,3$ - 39,3) entre 2012 e 2014 (Lessa et al., 2018) e evidenciam a influência da Água Tropical na BTS, a exemplo da Baía de Camamu, onde a intrusão da água da plataforma tropical também confere características oceânicas ao sistema (Affe et al., 2018). Essa influência costeira, fica evidente ainda na transparência na coluna d'água, que variou entre $3,0 \mathrm{~m}$ no dia $3(3,6 \pm 0,5 \mathrm{~m})$ e $7,0 \mathrm{~m}(6,5 \pm 0,5$ $\mathrm{m})$ no dia 1 (Figura 2D), diferindo significativamente entre os dias 1 e $2(p=0,0002)$ e os dias 1 e $3(p=$ 0,0009). Destacamos assim, a importância de estudos mais frequentes, incluindo análises de nutrientes inorgânicos dissolvidos, para possível prevenção destes eventos. 
Tabela 2. Estrutura da comunidade: variações da abundância $\left(\times 10^{4}\right.$ cél $\left.\mathrm{L}^{-1}\right)$, diversidade de Shannon-Wiener (bits.cel-1) e equabilidade de Pielou do microfitoplâncton total, registradas nos cinco pontos de coleta (P1, P2, P3, P4, P5), ao longo dos três dias de amostragem (25, 26, 27 agosto 2016) na Baía de Todos os Santos.

Table 2. Community structure: variations of total abundance $\left(\times 10^{4} \mathrm{~L}^{-1}\right)$, Shannon-Wiener Diversity (bits.cel $\left.{ }^{-1}\right)$ and Pielou equability of the total microphytoplankton recorded on the five collection points ( $P 1, P 2, P 3, P 4$, P5) over the three days of sampling $\left(25^{\text {th }}, 26^{\text {th }}, 27^{\text {th }}\right.$ August 2016) at Baía de Todos os Santos.

\begin{tabular}{|c|c|c|c|c|c|c|}
\hline \multicolumn{7}{|c|}{ Pontos } \\
\hline Dias & P1 & P2 & P3 & P4 & P5 & média ( $\pm \mathrm{DP})$ \\
\hline \multicolumn{7}{|c|}{ Abundância (Cél L-1) } \\
\hline 25 & 1,77 & 2,76 & 2,06 & 0,77 & 4,51 & $2,37( \pm 1,40)$ \\
\hline 26 & 1,84 & 1,01 & 2,19 & 1,24 & 2,18 & $1,71( \pm 0,55)$ \\
\hline 27 & 1,93 & 6,86 & 2,17 & 2,32 & 3,62 & $3,38( \pm 2,05)$ \\
\hline \multicolumn{7}{|c|}{ Diversidade (H') } \\
\hline 25 & 2,68 & 2,24 & 2,43 & 2,88 & 2,10 & $2,47( \pm 0,31)$ \\
\hline 26 & 2,64 & 2,21 & 2,85 & 2,43 & 3,06 & $2,64( \pm 0,34)$ \\
\hline 27 & 1,91 & 1,71 & 2,17 & 1,83 & 1,90 & $1,90( \pm 0,16)$ \\
\hline \multicolumn{7}{|c|}{ Equabilidade (J') } \\
\hline 25 & 0,87 & 0,72 & 0,84 & 0,74 & 0,81 & $0,80( \pm 0,06)$ \\
\hline 26 & 0,76 & 0,80 & 0,86 & 0,84 & 0,85 & $0,82( \pm 0,04)$ \\
\hline 27 & 0,67 & 0,67 & 0,67 & 0,63 & 0,63 & $0,66( \pm 0,02)$ \\
\hline
\end{tabular}

\section{AGRADECIMENTOS}

Ao Programa de Bolsas de Iniciação Científica (PIBIC) da Universidade Federal da Bahia. Esta pesquisa foi apoiada financeiramente pela Fundação de Amparo à Pesquisa do Estado da Bahia (FAPESB, no RED0006 / 2012). HMJA agradece a Coordenação de Aperfeiçoamento de Pessoal de Nível Superior - Brasil (CAPES - Finance Code 001) pela bolsa de doutorado. JMCN agradece ao Conselho Nacional de Desenvolvimento Científico e Tecnológico (CNPq \# 308542/2018-5) pela Bolsa de Produtividade em Pesquisa.

\section{BIBLIOGRAFIA}

Affe, H.M.J, Caires, T.A., Silva, E.M. \& Nunes, J.M.C. (2016). Floración de Trichodesmium erythraeum en la región costera tropical de Brasil. Revista de Biología Marina y Oceanografía, 51(1), 175-179. doi: 10.4067/S071819572016000100017.

Affe, H.M.J, Menezes, M. \& Nunes, J.M.C. (2018). Microphytoplankton in a tropical oligotrophic estuarine system: spatial variations and tidal cycles. Brazilian Journal of Botany, 41, 1-13. doi: 10.1007/s40415-018-0447-y.

Argôlo, N. \& Carmel, A. (2007). Sistema Estadual de Informações Ambientais da Bahia,2007. Assessoria de Comunicação Social, Centro de Recursos Ambientais (ASCOM/CRA). $<$ http://www.seia.ba.gov/notícias.cfm?idnoticia=3 266>

Attrill, M.J. \& Rundle, S.D. (2002). Ecotone or ecocline: ecological boundaries in estuaries. Estuarine Coastal Shelf Science, 55, 929-936.

Balech, E. (1988). Los dinoflagelados del atlántico sudoccidental. Instituto Español de Oceanografia, Plublicaciones especiales, Madrid, p 219.
Blauw, A.N., Beninca, E., Laane, R.W.P.M., Greenwood, N. \& Huisman, J. (2012). Dancing with the tides: fluctuations of coastal phytoplankton orchestrated by different oscillatory modes of the tidal cycle. PLOS ONE 11, 1-14. doi: 10.1371/journal.pone.0049319.

Brierley, A.S. (2017). Plankton. Current Biology Magazine 27, Scottish Oceans Institute, School of Biology, p 478-483.

Carvalho, M., Gianesella, S.M.F. \& SaldanhaCorrêa, F.M.P. (2008). Trichodesmium erythraeum bloom on the continental shelf off Santos, Southeast Brazil. Brazilian Journal of Oceanography, 56(4), 307-311.

Carvalho, R.C.Q., Cutrim, M.V.J., Eschrique, S.A., Azevedo-Cutrim, A.C.G., Moreira, E.G., Silveira, P.C.A. \& Coêlho, J.M. (2016). Microphytoplankton composition, chlorophyll-a concentration and environmental variables of the Maranhão Continental Shelf, Northern Brazil. Latin American Journal of Aquatic Research, 44, 256-266. doi: 10.3856/vol44-issue2-fulltext-7.

Castro, N.O. \& Moser, G.A.O. (2012). Florações de algas nocivas e seus efeitos ambientais. Oecologia Australis, 16(2), 235- 264. doi: 10.4257/oeco.2012.1602.05.

Cirano, M. \& Lessa, G.C. (2007). Oceanographic characteristic sof Baía de Todos os Santos,Brazil. Revista Brasileira de Geofísica, 25(4), 363-387.

Cloern, J.E. (1991). Tidal stirring and phytoplankton bloom dynamics in estuary. Journal of Marine Research, 49, 203-221.

Cloern, J.E., Foster, S.Q. \& Kleckner, A.E. (2014). Phytoplankton primary production in theworld's estuarine-coastal ecosystems. Biogeosciences, 11, 2477-2501. doi: 10.5194/bg-11-2477-2014 
Cupp, E.E. (1943). Marine plankton diatoms of the West coast of North America. University of California Press, London, p 237.

Falkowski, P.G. \& Raven, J.A. (2007). Aquatic photosynthesis. University Press, Princeton, $p$ 458.

Fernandes, L., Zehnder-Alves, L. \& Bassfeld, J. (2001). The recently established diatom Coscinodiscus wailesii (Coscinodiscales, Bacillariophyta) in Brazilian waters. I: Remarks on morphology and distribution. Phycological Research, 49, 89-96. doi: 10.1046/j.14401835.2001.00226.x.

Ferreira, A.N., Baretta, M. \& Mafalda, P.O.Jr. (2012). Avaliação do Impacto da dragagem sobre a associação fitoplanctônica do Porto de Aratu, Baía de Todos os Santos, Bahia. Arquivos de Ciências do Mar, 45(1), 30-46p.

Forte Neto, J.B., Baretta, M., Ferreira, A.N., Souza, C.S. \& Mafalda, P.O. Jr. (2014). A variabilidade da biomassa planctônica sob influência da sazonalidade e da dragagem do porto de Aratú, Baía de Todos os Santos, Brasil. Tropical Oceanography, 42(2), 230-242.

Gomes, D.F., Caldas, O., Silva, E.M., Gell, P.A. \& Williams, D.M. (2012). Father Zimmermann (1871-1950): the first Brazilian diatomist. Diatom Research, 27(3), 177-188.

Hatje, V., Andrade, J.B. (2009). Baía de Todos os Santos: Aspectos Oceanográficos. Salvador: EDUFBA, 304p.

Hydros (2005). Análise preliminar de risco à saúde humana. Relatório síntese, $34 \mathrm{p}$.

Lessa, G.C., Cirano, M., Genz, F., Tanajura, C.A.SO \& Silva, R.R. (2009). Oceanografia Física. In: Hatje, V, Andrade, JB. (Org.) Baía de Todos os Santos: Aspectos Oceanográficos. Salvador: EDUFBA, p. 71-119.

Lessa, G.C., Souza, M.F.L., Mafalda, P.O. Jr, Gomes, D.F., Souza, C.S., Teixeira, C.E.P., Souza, J.R.L.B. \& Zucchi, M.R. (2018). Variabilidade intra-anual da oceanografia da Baía de Todos os Santos: evidências de três anos de monitoramento. In: Hatje, V., Dantas, L.M.V., Andrade, JB. (Org.) Baía de Todos os Santos: avanços nos estudos de longo prazo. Salvador: EDUFBA, p. 159-192.

Lopes, R.M., Dias, J.F. \& Gaeta, A.S. (2009). Ambiente Pelágico. In: Hatje, V., Andrade, J.B. (Org.) Baía de Todos os Santos: Aspectos Oceanográficos. Salvador: EDUFBA, p. 123-155.

Medeiros, C., Macedo, S.J., Feitosa, F.A.N. \& Koening, M.L. (1999). Hydrography and phytoplankton biomass and abundance of NorthEast Brazilian waters. Archive of Fishery and Marine Research, 47, 133-151.

Moestrup, Ø., Akselman, R., Cronberg, G., Elbraechter, M., Larsen, J., Lundholm, N., Nguyen, L.N. \& Zingone, A. (2009). IOCUNESCO Taxonomic reference list of harmful microalgae. <http://www.marinespecies.org/HAB>.

Proença, L.A.O., Tamanaha, M.S. \& Fonseca, R.S. (2009). Screening the toxicity and toxin of blooms of the Cyanobacterium Trichodesmium erythraeum (Ehrenberg) in Northeast Brazil. Journal of Venomous Animals and Toxins including Tropical Diseases, 15(2), 204-215.

Proença, L.A.O., Fonseca, R.S. \& Pinto, T.O. (2010). Microalgas em área de cultivo do litoral de Santa Catarina. Pp. 331-331. In: XIII Congresso Brasileiro de Ficologia. Paraty, RJ, Brasil (Anais).

Reguera, B., Riobó, P., Rodríguez, F., Díaz, P.A., Pizarro, G., Paz, B., Franco, J.M. \& Blanco, J. (2014). Dinophysis Toxins: Causative Organisms, Distribution and Fate in Shellfish. Marine Drugs, 12(1), 394-461. doi: 10.3390/md12010394.

Reynolds, C.S. (2006). The ecology of phytoplankton. Cambridge University Press, Cambridge, p 507.

Rezende, K.R.V., Hatherly, M.M.F., Pimenta, C.M.M., Eduardo, J., Vianna, S.C. \& Mangiavacchi, N. (2015). Phytoplankton community structure in one sector of Guanabara Bay (RJ, Brazil) during 2011 and 2012. Brazilian Journal of Oceanography, 63, 239-254.

Santana, R, Teixeira, C, Lessa, G. (2018). The Impact of Different Forcing Agents on the Residual Circulation in a Tropical Estuary (Baía de Todos os Santos, Brazil). Journal of Coastal Research, 34(3), 544-558. doi: 10.2112/JCOASTRES-D-17-00044.1

Saraiva, JAP. (2008). Baia de Todos os Santos: vulnerabilidades e ameaças. Dissertação (Mestrado). Universidade Federal da Bahia. Escola Politécnica. Salvador, 2008. 191p.

Satô, S., Paranaguá, M.N. \& Eskinazi, E. (1966). On the mechanism of red tide of Trichodesmium in Recife northeastern Brazil, with some considerations of the relation to the human disease 'Tamandare fever'. Trabalhos do Instituto Oceanografico da Universidade do Recife, 5, 749.

SEAP (2007). Relação de organizações. Secretaria Especial de Aquicultura e Pesca - SEAP. Subsecretaria de Desenvolvimento de Aquicultura e Pesca - Gerência de Cooperativismo - GECOOP. Presidência da República, $455 \mathrm{p}$.

Siqueira, A., Kolm, E.H. \& Brandini, F.P. (2006). Offshore distribution patterns of the cyanobacterium Trichodesmium erythraeum Ehrenberg and associated phyto- and bacterioplankton in the Southern Atlantic coast (Paraná, Brazil). Brazilian Archives of Biology and Technology, 49(2), 323-337.

Soares, L.S.H., Lopez, J.P., Muto, E.Y. \& Giannini, R. (2011). Capture fishery in theNorthern Todos os Santos Bay, Tropical Southwestern Atlantic, Brazil. Brazilian Journal of Oceanography, 59(1), 61-74.

Souza, C.S., Oliveira, O.C., Mafalda, P.O.Jr. \& Marcolin, C.R. (2008). Caracterização de eventos de floração nociva provocada por Gymnodinium sanguineum na Baía de Todos os Santos, Salvador, BA. In: Anais do I congresso IberoAmericano de Oceanografia, Fortaleza, 2008. 
Throndsen, J., Hasle, G.R. \& Tangen,, K. (2007). Dinoflagellates-Dinophyta. In: Throndsen J, Hasle, GR, Tangen, K (eds) Phytoplankton of Norwegian coastal Waters. AlmaterForlag AS, Oslo, Norway, pp 41-110.

Tomas, CR. (1997). Identifying marine phytoplankton. Florida Academic Press, Miami, p 858.
Utermöhl, H. (1958). Zur Vervolkommung der quantitativen phytoplankton-methodik. Mitt Int Ver Theor Angew Limnol, 9, 1-38.

Wolgemuth, K., Burnett, W. \& Moura, P.L. (1981). Oceanography and suspended materials in Todos os Santos Bay. Revista Brasileira de Geociências, 11, 172-178. 